\title{
Patient participation in the development, delivery of health care services, and implications for quality: Protocol for a scoping review.
}

\author{
Authors and affiliation \\ Joachim Sagen 1, 2, Geir Smedslund ${ }^{1,}$ Ingvild Kjeken ${ }^{1,2,}$ Hanne Dagfinrud ${ }^{1,}$ Andreas Habberstad ${ }^{3,}$ Ann \\ Elisabeth Simonsen ${ }^{4}$ Rikke Helene Moe ${ }^{1}$
}

1) National Advisory Unit in Rheumatology.

2) Oslo Metropolitan University.

3) The Norwegian Federation of Organisations of Disabled People.

4) Patient representative at Røysumtunet rehabilitation center.

\section{Registration}

The final protocol was registered at Open Science Framework and ResearchGate

DOI: 10.31219/osf.io/ysa9v

\section{Update}

At the end of the introduction section we have clarified the aim of the review. In the rationale and clarification of concept section we have extended the reasons for performing the scope. As part of the objective section, we have added the study purpose and clarified research question three. In the section about identifying relevant studies we have extended the eligibility criteria.

\section{Introduction}

Historically, there has been an expectation that patients are passive recipients of health care services, but now we are moving towards a more participatory health care approach. Over the last few decades, patient participation has been increasingly advocated by stakeholders in and beyond the healthcare system. Patient participation is about patients, relatives and patient representatives being part of the collaboration with professionals at different levels of care. Potentially, patients can contribute to achieving the best possible treatment. Patient participation is nevertheless still not always an incorporated part of the work routines, neither in individual patient treatment (microlevel), in institutional decisions (meso level) nor in decisions on a governmental level (macro level) [1]. This scoping review will therefore summarize existing knowledge about patient participation in the development and delivery of health care services at meso level. The aim is to increase the understanding of current experiences, knowledge, motives, actions, behaviors and outcomes at the meso level.

\section{Rationale}

Patient participation is increasingly important in the development and delivery of health care services, particularly when it is performed in a good manner [2]. Patient participation can contribute to better quality in health care services. It is clearly stated that patients should have a central position in their own healthcare process [3]. As far back as to the World Health Organization's Declaration of Alma Ata in 1978 it was stated that "people have the right and duty to participate individually and collectively in the planning and implementation of their health care" [4]. Over the last years there has been increasing attention to patient participation in the design and implementation of healthcare services, but there is limited agreement on how to make it happen [5]. There is conflicting evidence about the impact of involving patients in health service development. 
High quality evidence on implications about the development, delivery and quality of care is warranted [6-10]. Nevertheless, a recent scoping review investigating impacts of patient participation at a meso level found that different types of patient participation (e.g. collaborative working groups, patients councils etc.) give positive outcomes for health service development (e.g. better organization's communication, decreased hospital admissions and reduced costs etc.) [11]. Another scoping review identified the following meso level characteristics that could support shared decision making at the micro level: incentives, policies and guidelines, culture of healthcare delivery, and healthcare provider education and licensing [12]. This scoping review will summarize existing knowledge about patient participation and the impact on health care services at the meso level. We will map and describe characteristics of meso level approaches to patient participation and facilitators for successful patient participation. As an extension to the previous scooping reviews, we will examine context as a facilitating factor for meso level patient participation.

\section{Clarifications of concepts}

Several terms have been used in the literature to describe the involvement of patients at different levels of the health care service [13]. In this review we will use the generic term patient participation when referring to this interaction. Implementing and evaluating patient participation is a complex process including several concepts and dimensions. Patient participation involves patients, healthcare professionals, healthcare institutions and government. In this review we will use a proposed conceptual model of patient participation where dimensions of patient participation are divided into a micro, meso and macro level [13] . Patient participation includes shared decision making by involving patients in decisions about their own individual care (micro level), as patient representatives regarding health care service decisions at institutional level (meso level) and as patient representatives at a societal and governmental level (macro level). This scoping review will systematically map and identify existing gaps in knowledge to inform future research, clarify key concepts and examine emerging evidence by addressing patient participation at the meso level.

\section{Strengths and limitations of this study}

\section{Strengths}

- A broad scoping review can serve as useful information for health authorities, health organizations, health professionals, patient organizations and others working within or conducting research in this area.

- The results can contribute to generating guidelines for implementing patient participation at the meso level.

\section{Limitations}

- Rather than selecting only the highest quality of evidence, a scoping review attempts to describe all the information available. The included studies will therefore not undergo a formal quality assessment.

- This protocol is for a scoping review that only considers material written in English, Swedish, Danish or Norwegian. Potentially, large populations of the non-English or non-Nordicspeaking world may not be represented. Our conclusions may not apply to the different cultural and social environments in these regions.

\section{Methods}

Our protocol was drafted using the preferred reporting items for systematic reviews and metaanalysis protocols (PRISMA - P) [14] as far as applicable to the scoping review methodology. 
To address the aims of the review we will conduct a systematic scooping review. This review will be guided by Arksey and O'Malley's framework [15] as well as by the recommendations by Levac [16]. They recommend organizing the review process in six stages: 1 . Identifying the research question, 2 . Identifying relevant studies, 3 . Study selection, 4 . Charting the data, 5 . Collating, summarizing and reporting the results and 6 . Consider potential considerations to develop advancement, application and relevance of scoping studies in health research.

\section{Stage 1: Identifying the research question}

\section{Objective}

The present study undertakes a scoping review of research on patient participation in the development and delivery of health care services and implications for quality, following the PRISMA checklist for scooping reviews [17]. The purpose is to gain extended insight into factors that may influence patient participation, including barriers and facilitators with the potential to affect quality.

Because of the broadly defined research purpose, we will identify key elements by using frameworks such as (but not limited to) PCC: Population, Concept, and Context. Population criteria: Patients and healthcare professionals in healthcare services (16 years and above). Concept criteria: Patient participation in development and delivery of healthcare services. Context criteria: Practices, motives and outcomes. A scoping review will be conducted in order to systematically map the research in the area, identify existing gaps in knowledge, as well as to report on types of evidence. The objective will be guided by the following research questions:

1. How are stakeholders like patient representatives, health care professionals and organizations involved in service development and delivery?

2. What are the reported results when involving patient representatives in the development and delivery of health care services?

3. What facilitates and hinders successful patient participation at the meso level in health care services?

4. What characterizes meso level approaches to patient participation?

\section{Stage 2: Identifying relevant studies}

\section{Eligibility criteria}

Relevant studies that match the review questions and are appropriate for the objectives will be considered for inclusion. All primary studies in English, Norwegian, Swedish or Danish language will be eligible. The participants will be patients or healthcare professionals in healthcare services.

Studies focusing on persons older than 16 years old are considered appropriate for inclusion. Studies will be excluded if study participants because of their condition alone cud be a constraint on autonomy, communication and cooperation skills (e.g., studies involving people with serious mental illness, cognitive dysfunction or end of life care). Studies exploring patient participation in research, education or the development of instruments or tools will not be eligible. Final study selection and eligibility criteria will be developed post hoc based on increased familiarity with the literature.

\section{Stage 3: Selecting studies}

\section{Information sources}

To identify potentially relevant documents, we will do a comprehensive search in the following bibliographic databases: MEDLINE, EMBASE, Cochrane and PsycINFO. The search strategies will be refined through team discussion and further drafted by an experienced librarian. We will also 
conduct a thorough search of grey literature. The search for documents in the grey literature can include electronic sources such as; OpenSIGLE, Healthcare Management Information Consortium (HMIC), GoogleScholar, PsycEXTRA, Open Grey, and OpenThesis. Reference lists of identified reviews, reports and articles will also be searched for additional studies.

\section{Search strategy}

The final search results will be exported into EndNote and from EndNote to Covidence and screened for duplicates. After duplicates are removed the remaining search will undergo a title and abstract screening. When inclusion or exclusion cannot be determined on the basis of title and abstract, the person screening the item will move the item forward for full-text screening. Three reviewers will be required for full text screening. The search strategy that has been used for Cochrane, search words, including planned limitation is available in Supplement 1.

\section{Stage 4: Charting the data}

The data charting form will be developed by one team member, then pilot tested by two team members using six included studies. The form will be revised to reach consensus on the data collection process and to add additional categories. The charting form will be updated if necessary. We will both extract general information on each study and specific information related to quality indicators for successful health care delivery. The search decision process will be described using the PRISMA flow diagram [17].

\section{Stage 5: Collating, summarizing and reporting the results}

We will conduct a descriptive numerical analysis of characteristics of the included studies (study designs, years of publication etc.), as well as a qualitative thematic analysis of the meso level factors identified in the studies. The results of this thematic analysis will be reported in a tabular form with a list of factors. Furthermore, the meaning of the results will be discussed narratively in relation to the study objectives.

\section{Patient and public participation}

Two patient representatives have been a part of the research team from the very first beginning, they clarified the importance of the research topic and initiated the study. Patient representatives participated in developing the project plan and study design. They will assist interpret the findings, prepare the manuscript, and disseminate the results to patient organizations and decision makers.

\section{Ethics and dissemination}

A scoping review does not require ethical approval. Results will be disseminated through academic journals, conferences and seminars for health care professionals, patient organizations, included health care institutions, and communicated to policymakers.

\section{Funding and support}

The project is funded by the Dam Foundation. Dam is one of Norway's largest foundations and provides support to health and research projects. 


\section{References}

\section{Primary Sources}

1. Solbjør, M. and A. Steinsbekk, [Patient participation in hospital wards--health personnel's experience]. Tidsskr Nor Laegeforen, 2011. 131(2): p. 130-1.

2. Coulter, A., et al., Shared decision making in the UK: Moving towards wider uptake. Z Evid Fortbild Qual Gesundhwes, 2017. 123-124: p. 99-103.

3. EuropeanCommission, Eurobarometer Qualitative Study, PATIENT INVOLVEMENT, Aggregate Report 2012, European Commission, Directorate-General for health and Consumers

4. WHO, Declaration of Alma Ata, in Conference on Primary Health Care. 1978, World Health Organization: Geneva. p. 6-12.

5. Dukhanin, V., R. Topazian, and M. DeCamp, Metrics and Evaluation Tools for Patient Engagement in Healthcare Organization- and System-Level Decision-Making: A Systematic Review. Int J Health Policy Manag, 2018. 7(10): p. 889-903.

6. Nilsen, E.S., et al., Methods of consumer involvement in developing healthcare policy and research, clinical practice guidelines and patient information material. Cochrane Database Syst Rev, 2006. 2006(3): p. Cd004563.

7. Mockford, C., et al., The impact of patient and public involvement on UK NHS health care: $a$ systematic review. Int J Qual Health Care, 2012. 24(1): p. 28-38.

8. Crawford, M.J., et al., Systematic review of involving patients in the planning and development of health care. Bmj, 2002. 325(7375): p. 1263.

9. Fønhus, M.S., et al., Patient-mediated interventions to improve professional practice: $A$ summary of a Cochrane systematic review. Patient Educ Couns, 2019. 102(3): p. 474-485.

10. Fønhus, M.S., et al., Patient-mediated interventions to improve professional practice. Cochrane Database Syst Rev, 2018. 9(9): p. Cd012472.

11. Sandvin Olsson, A.B., et al., How can we describe impact of adult patient participation in health-service development? A scoping review. Patient Educ Couns, 2020. 103(8): p. 14531466.

12. Scholl, I., et al., Organizational-and system-level characteristics that influence implementation of shared decision-making and strategies to address them - a scoping review. Implement Sci, 2018. 13(1): p. 40.

13. Halabi, I.O., et al., "Patient participation" and related concepts: A scoping review on their dimensional composition. Patient Educ Couns, 2020. 103(1): p. 5-14.

14. Moher, D., et al., Preferred reporting items for systematic review and meta-analysis protocols (PRISMA-P) 2015 statement. Syst Rev, 2015. 4(1): p. 1.

15. Arksey, H. and L. O'Malley, Scoping studies: towards a methodological framework. International Journal of Social Research Methodology, 2005. 8(1): p. 19-32.

16. Levac, D., H. Colquhoun, and K.K. O'Brien, Scoping studies: advancing the methodology. Implement Sci, 2010. 5: p. 69.

17. Tricco, A.C., et al., PRISMA Extension for Scoping Reviews (PRISMA-SCR): Checklist and Explanation. Ann Intern Med, 2018. 169(7): p. 467-473. 
Supplement 1: Cochrane, search words, including planned limits

Database: Ovid MEDLINE(R) and Epub Ahead of Print, In-Process \& Other Non-Indexed Citations and Daily $<1946$ to July $06,2020>$

Search Strategy:

1 exp Patient Participation/ (25749)

2 exp Health Planning/ (346770)

3 exp Health Policy/ (106014)

4 exp "Delivery of Health Care"/ (1074715)

5 exp Organizational Policy/ (14096)

6 exp Health Services/ (2128674)

7 exp Decision Making, Organizational/ (11109)

8 exp Decision Making/ (201321)

9 user engagement.mp. (436)

10 involvement.mp. (451499)

11 representatives.mp. (25706)

12 committees.mp. (33831)

13 Quality.mp. (1191129)

14 delivery.mp. (579119)

15 advisory councils.mp. (98)

162 or 3 or 4 or 5 or 6 or 7 or 8 or 9 or 10 or 11 or 12 or 13 or 14 or 15 (4671220)

171 and $16(25749)$

1817 and 2020:2020.(sa_year). (94)

19 limit 18 to (danish or english or norwegian or swedish) (87)

20 limit 19 to ("young adult (19 to 24 years)" or "adult (19 to 44 years)" or "young adult and adult (19-

24 and 19-44)" or "middle age (45 to 64 years)" or "middle aged (45 plus years)" or "all aged (65 and over)" or "aged (80 and over)") (45)

21 exp Primary Health Care/ (158760)

22 exp General Practitioners/ (7862)

$2320 \operatorname{not}(21$ or 22$)(36)$ 